\title{
The Distance Learning course on Computer Networks at the University of Coimbra
}

\author{
João Costa e Silva, Sandra Pedrosa, Maria José Marcelino, António Mendes, Fernando \\ Boavida, Mário Bernardes
}

\begin{abstract}
: this article presents a description and characterization of the first edition of the course on Computer Networks promoted by the Special Project for Distance Learning of the University of Coimbra. It establishes a brief comparative analysis of the course on Computer Networks and other similar courses coming up with some conclusions that can be useful for a better understanding of the strategies for planning and developing the course on future editions.
\end{abstract}

Key words: Computer networks; Distance Learning; Lifelong learning; Moodle; LMS

\section{INTRODUCTION}

In the past few years we have witnessed a deep change in the society's behavior. The appearance of satellites, television, computers, Internet, optic fiber, among many other innovations, has allowed the development of a society, as we know it today. Beyond the obvious advantages brought to our daily lives.

The development of ICT (Information and Communication Technologies) brought also changes in education in general and allowed the emergence of new models of teaching and learning.

In the area of Distance Learning (DL), we have been witnessing an academic and professional revolution regarding education and training. This fact increasingly inflicts the need of improving education and training to cover exclusion when the educational and professional profiles become out-of-date [Bates, 2005]. The continuous need for updating people's knowledge calls for DL methodologies, as they give the time and place flexibility that working people need.

The Distance Learning Project of the University of Coimbra (UC_D) was created to address continuous education needs of active people. It is based in the "knowledge and in the experience of the UC teachers of several areas of knowledge" [UC_D, 2014]. The project started in late 2010 and is supported by a multi-disciplinary team with competences in instructional design, multimedia and interface design, and informatics. There are also scientific, pedagogic, and administrative coordination teams.

The project team works in close cooperation with the teachers in several tasks related with the design of distance learning courses: definition of pedagogic strategies to use, multimedia materials development, interface design, and so on.

UC_D has developed several courses in different areas, such as: Life Sciences; Social Sciences; Engineering; Management; Economics; Humanities; Law; Sports

In this paper we will describe the course on Computer Networks, including the results of a satisfaction inquiry carried out after its first edition.

\section{RELATED WORK}

Nowadays the Internet allows an easy access to educational content, such as

\footnotetext{
Permission to make digital or hard copies of all or part of this work for personal or classroom use is granted without fee provided that copies are not made or distributed for profit or commercial advantage and that copies bear this notice and the full citation on the first page. Copyrights for components of this work owned by others than ACM must be honored. Abstracting with credit is permitted. To copy otherwise, or republish, to post on servers or to redistribute to lists, requires prior specific permission and/or a fee. Request permissions from Permissions@acm.org.

CompSysTech'14, June 27-28, 2014, Ruse, Bulgaria.

Copyright (C2014 ACM ISBN 978-1-4503-2753-4/14/06...\$15.00.

http://dx.doi.org/10.1145/2659532.2659640 
books and magazines in digital format; technical and scientific papers; blogs, social networks, basic and advanced training courses that may or may not give a degree, among others [Prensky, 2004]. This allows learners to select what suits them best. The training that has always existed in classroom settings appears now also in the form of distance education through e-learning and b-learning.

In the area of computer networks it is also possible to find several courses, both provided by high education institutions and some specialized companies. Well know examples are those provided by CISCO, allowing participants to get certifications, such as the CCENT (Cisco Certified Entry Networking Technician) and CCNA (Cisco Certified Network Associate) [CISCO, 2014].

In some cases, these courses use simulators with a didactic purpose, such as the Network Simulator Packet Tracer CISCO. Using those tools students are virtually in contact with work tools. This would be problematic to be done in real settings, both due to access difficulties and also due to the infrastructure costs involved [Pinheiro et al., 2009]. So, technology is used to give access to knowledge, but also to improve the learning experience.

The access to knowledge can be fostered through learning based on new technologies, namely e-learning platforms, responding to a training and practice need. This happens especially if taking into consideration the general difficulty of countless professionals that can attend training outside their normal working hours regime. [Magano, Sochirca, Carvalho, 2009].

\section{COURSE DESCRIPTION}

The course on Computer Networks offered by the University of Coimbra Distance Learning Project was developed in partnership with the Informatics Engineering Department of the Faculty of Sciences and Technology. It offers a theoretical framework to practical tasks of equipment configuration, traffic analysis and identification of damages in TCP / IP local area networks (LANs), using open-source code tools available in common operating systems. The main goals are to enable trainees to configure computers with Linux, Apple Mac OSX or Microsoft Windows operating systems for their integration in TCP / IP networks, to verify the connectivity between equipment, to diagnose problems using traffic analysis tools and to understand and verify the operation of network support applications (DNS and DHCP).

The course has an estimated 54h workload and doesn't include any presential or synchronous sessions, giving total place and time flexibility to students. It is conducted by two very experienced teachers who are specialists on computer networks. The target audience for the course includes people who completed secondary education, but also persons who have some university education, namelly in Engineering and Science.

To allow a gradual learning, the course has a sequential modular structure, with specific goals, contents, activities and assessments in each module. Each module has its own schedule, with specific starting and closure dates.

This course is based on individual study and work, assuming that the learner actively explores the available materials. The trainee is also encouraged to share his/her doubts and difficulties with peers and teachers, promoting in this way a deepening and broadening of the class points of view.

Knowledge acquisition is based on theoretical and practical materials, available throughout each module, in various formats, such as texts with concepts presentation, explanatory videos demonstrating procedures, and interactive diagrams. The learner can also use a set of tools (fora, messaging) that may support the learning construction process. Fora are seen as collaborative learning tools, where learners can share experiences, ask questions and become engaged in building a learning and practice community. To facilitate student integration in the course, the first two days of the course 
are dedicated to welcome and familiarization activities, such as the presentation of each participant to the group and an integration forum where they are supposed to share their expectations about the course. Throughout the course there is a general forum where learners can discuss general topics under the theme, launch discussions, share experiences and post general questions. For each module, there is also a specific forum where questions can be placed about the module in question.

The course consists of five modules: Module 1 | Basic Concepts, Module 2 | Local Area Networks (LAN), Module 3 | TCP / IP Architecture, Module 4 | Addressing and Routing and Module 5 | Internet Naming System (DNS).

In Module 1 the main objective is to provide an introduction to computer networks and basic concepts necessary to study the transmission of information. In addition to presenting basic notions of representation and transmission of information in a computer network, the main modes of data transmission, the concept of multiplexing, network topologies, and circuit and packages switching networks are described.

Module 2 studies local computer networks which, in most cases, are used to connect personal computers and application servers. The key technologies of local networks are presented and physical aspects implementation is addressed.

An overview of the TCP / IP architecture is presented in Module 3. It starts by studying a layered communications architecture representation, by presenting and comparing models of the OSI and the TCP / IP. This approach allows understanding the architecture of the Internet communication model and contributes to a future more structured and methodical practical approach. It is followed by an overview of the TCP / IP architecture layers and an identification of its main protocols. Finally, the major types of applications are also identified.

Module 4 examines the main aspects necessary for configuring IP connectivity: equipment addressing and routing between networks. The concepts of address and subnet mask are presented, as well as representation alternatives. The organization of the address space is described and the concepts of subnetwork and supernetwork are introduced. The use of public addresses and the need to use the private address space instead of public addresses are also discussed. The module ends with an introduction to static and dynamic Internet routing, with its basic principles, the architecture of Internet routing, and the main types and routing protocols.

Finally, Module 5 introduces the Internet naming system, called Domain Name System (DNS), one of the most critical applications to support the performance of computer networks. However, DNS is also one of the less known applications and it often disturbs the use of other Internet applications and unease's problems diagnosis. The module describes the space naming and the implementation of the DNS application using a hierarchy of name servers. The module includes tools that allow to apply the concepts presented and to validate the operation of the DNS.

Each module includes also consolidation questions and/or practical exercises that can be done in a residential network (domestic). There are several formative and optional activities that are available in each topic (figure 1). At the end of each module, several assessment exercises are proposed to participants. These include activities to be carried on the trainees' equipment and access networks (e.g. RFCs analysis and identification of addresses and domains). 


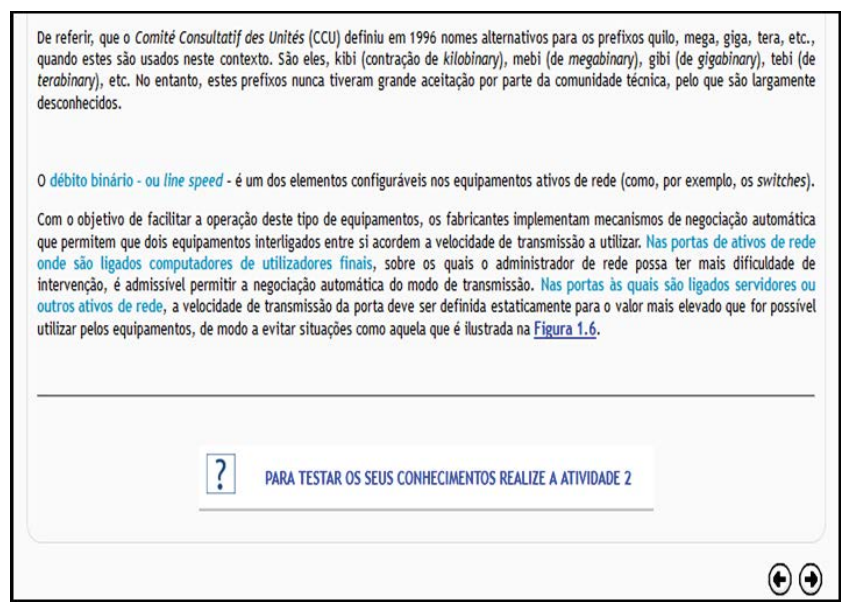

Figure 1 - Formative and optional activities available over the topics

\section{INSTRUCTIONAL DESIGN AND MONITORING OF THE COURSE}

The course development started in a meeting between the teachers and the project instructional design team. The course themes, goals, target-public, workload, program structure, among other items were defined. The meeting was also used for a first definition of the course graphical image and main guidelines of the presentation video that would be recorded by the professors.

In a second phase, a working plan was created. It included a schedule (based on the distribution of the workload), a list of activities and support resources.

Finally, in a third phase, the contents produced by the teachers and multimedia materials produced by the project team were integrated in meaningful educational resources that were organized in the LMS used for the course. Texts and images were used to present and explain concepts. Interactive diagrams and explanatory videos were also used when necessary to a proper understanding of the concepts involved (figure 2).
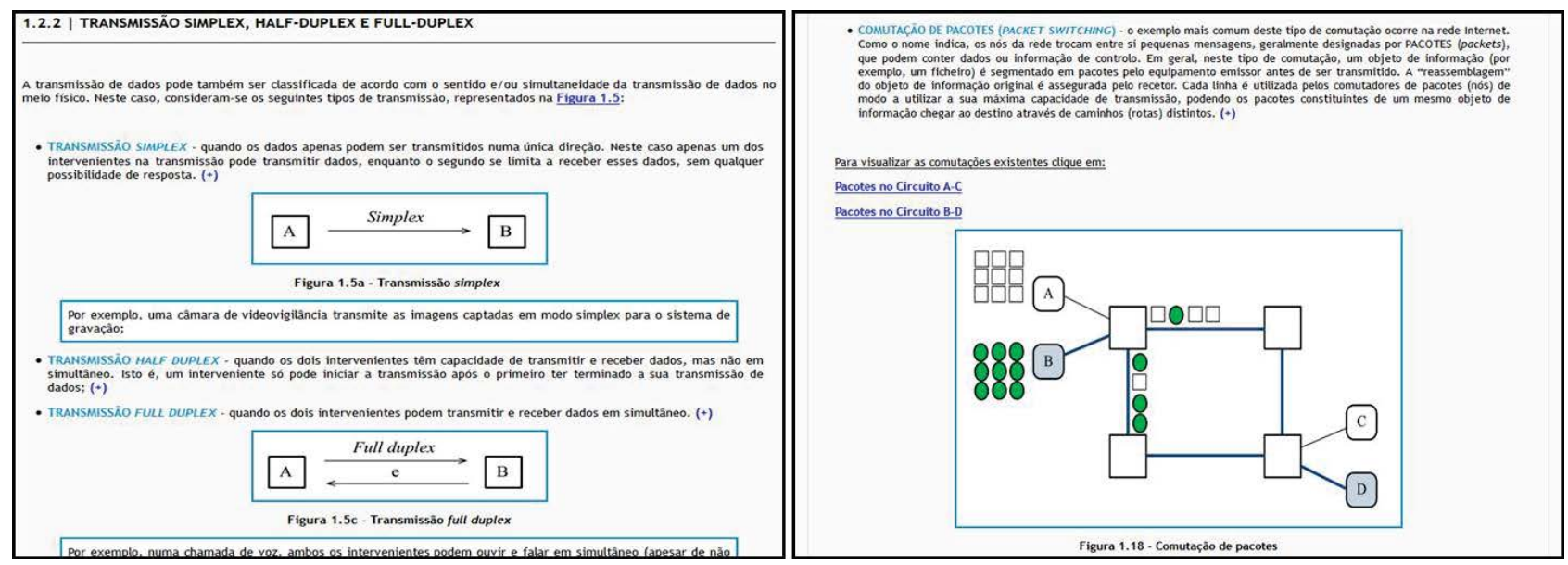

Figure 2 - Examples of content outlined and organized in several formats

Two types of videos were produced: Videos in which the teacher was filmed explaining a particular process, and screen capture based videos in which the teacher explained a certain process (figure 3 ). 

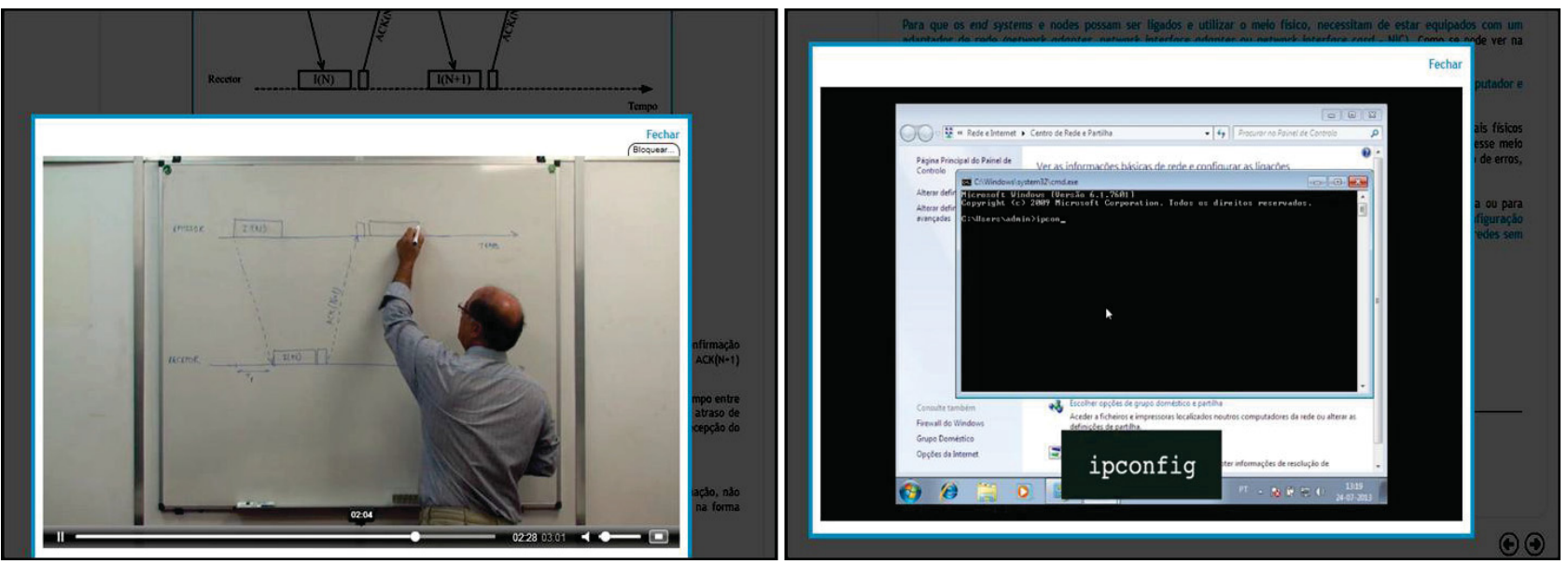

Figure 3 - Types of videos produced

The assessment activities were supported by the LMS file submission facility. To facilitate the participants' work, the team prepared templates in Word (docx), and in OpenDocument Text (odt). Those templates include all information necessary for the task, and also space for the participants' input. So, they had to download the template, do the required activity, and upload the file again to the LMS. Teachers could later make comments on the formative activities and correct and grade the assessment ones. teachers.

Before the course started all the contents and activities had to be validated by the

During the course, the work of the learners was monitored daily. Teachers answered questions and promoted discussions, while the project team solved some minor technical difficulties and helped teachers to keep track with all the work necessary.

\section{COURSE SATISFACTION ASSESSMENT}

The first edition of the course took place between October 08th and December $11^{\text {th }}$ 2013. It had 9 participants. When the course closed, a satisfaction questionnaire was made available on the platform, in order to evaluate the satisfaction of the participants regarding the course and all the involved individuals.

The questionnaire had the following categories: usability of the platform, course content, multimedia resources, bibliography, teaching team, interpersonal relationships, expectations satisfaction, level of knowledge, strengths and weaknesses, suggestions and recommendations.

The outcome of this questionnaire, completed by 7 trainees, allowed us to conclude that most of them expressed satisfaction with the first edition of the course on Computer Networks of the University of Coimbra.

Almost all categories were positively assessed by trainees, including: the interest and importance of the topic addressed, the quality of the course contents and planned activities, the monitoring carried out by the teaching staff and teaching resources available. The learning platform used was also mentioned as a positive aspect.

However no category reached full agreement from all the trainees. This shows that there are always features that can and should be improved. In terms of suggestions made by students, we highlight the areas for improvement in future editions of this course: changing the deadlines for carrying out the activities, the development of some topics and supporting materials, the strengthening of the practical component through more practical examples, an higher interaction between participants and more activities' feedback. 


\section{CONCLUSIONS AND FUTURE WORK}

"The current situation of e-learning is of development and evolution. There are a number of initiatives at all levels, from teaching to technological aspects. At the pedagogical level, we seek to identify strategies for teaching/learning that best suit specific cases.

Above all, e-learning should be seen as a different educational tool, with its potential, difficulties and restrictions that are inherent to it. The investment of HEl [Higher Education Institutions] in e-learning should be well integrated into an extension concern with improved processes of teaching and learning, rather than being a strategic objective concern per se." [Magano, Sochirca, Carvalho, 2009, p. 24].

In this paper we described and present the main results of the first edition of the distance-learning course on Computer Networks of the University of Coimbra.

At the moment, the second edition of the course is taking place. Some changes have already been made in consequence of the results of the satisfaction evaluation of the first edition of the course. These changes include making all the activities mandatory in order to keep participants more time in the platform and therefore improve their efficiency and interaction with colleagues.

As future work we will consider a new b-learning version of the course, including a strong practical component.

\section{REFERENCES}

[1] A. Bates. (2005). Technology, E-Learning and Distance Education (2nd edition), London: Routledge. [Online]. Available: http://www.google.pt/books?id=_N2CAgAAQBAJ\&dq=BATES,A.+\%282005\%29:+\%E2\%8 0\%9CTechnology,+E-

Learning+and+Distance+Education $\%$ E2\%80\%9D,+2nd+edition,+London:+Routledge.\&lr= \&hl=pt-PT

[2] CISCO. (2014). Training \& Certifications. [Online]. Available: http://www.cisco.com/web/learning/certifications/index.html

[3] J. Magano. E. Sochirca. C. V. Carvalho. (2009). O e-learning como factor de sucesso gestão da inovação. [Online]. Available: http://www.iscet.pt/sites/default/files/Percursosldeias/N_1/Revista2009.pdf

[4] R. P. Pinheiro, F. A. A. Lins, J. C. B. Melo. A Utilização de Simulação no Ensino de Redes de Computadores. [Online]. Available: http://www.eventosufrpe.com.br/jepex2009/cd/resumos/R0311-1.pdf

[5] M. Prensky. (2004). The Emerging Online Life of the Digital Native. [Online]. Available: http://www.marcprensky.com/writing/Prensky-

The_Emerging_Online_Life_of_the_Digital_Native-03.pdf

[6] UC_D (2014). Redes de Computadores (1st edition). [Online]. Available: http://www.ed.uc.pt/educ/curso?id=27

\section{ABOUT THE AUTHORS}

João Costa e Silva, Master, Distance Learning Project, University of Coimbra, Phone: +351 239242717, E-mail: j.silva@uc.pt

Sandra Pedrosa, Master, Distance Learning Project, University of Coimbra, Phone: +351 239242717, E-mail: sandra.pedrosa@uc.pt

Maria José Marcelino, PhD, Department of Informatics Engineering, University of Coimbra, Phone: +351 239 790067, E-mail: zemar@dei.uc.pt

António José Mendes, PhD, Department of Informatics Engineering, University of Coimbra, Phone: +351 239 790036, E-mail: toze@dei.uc.pt

Fernando Boavida, PhD, Department of Informatics Engineering, University of Coimbra, Phone: +351 239 790000, E-mail: boavida@uc.pt 
Mário Bernardes, ICT Director, University of Coimbra, Phone: +351239 242880, Email: mjb@uc.pt 